\title{
Dynamic Alterations of Anti-S-Protein Igg Subclasses and of Th1/Th2 Responses are Hallmarks of Acute Severe COVID-19 Disease
}

\section{Yun Shan Goh}

Technology and Research (A*STAR)

\section{Siew-Wai Fong}

Technology and Research (A*STAR)

Siti Naqiah Amrun

Technology and Research (A*STAR)

Cheryl Lee

Technology and Research (A*STAR)

Pei Xiang Hor

Technology and Research (A*STAR)

\section{Barnaby Young}

National Centre for Infectious Diseases, 16 Jalan Tan Tock Seng

Po Ying Chia

National Centre for Infectious Diseases, 16 Jalan Tan Tock Seng

Paul Tambyah

National Centre for Infectious Diseases, 16 Jalan Tan Tock Seng

Shirin Kalimuddin

Singapore General Hospital

\section{Surinder Pada}

$\mathrm{Ng}$ Teng Fong Hospital

Seow-Yen Tan

Changi General Hospital

\section{Louisa Sun}

Alexandra Hospital

\section{Mark Chen}

National Centre for Infectious Diseases, 16 Jalan Tan Tock Seng

\section{Yee-Sin Leo}

National Centre for Infectious Diseases, 16 Jalan Tan Tock Seng

\section{David Lye}

National Centre for Infectious Diseases, 16 Jalan Tan Tock Seng

Lisa $\mathrm{Ng}$ 
Technology and Research (A*STAR)

Laurent Renia ( $\sim$ renia_laurent@IDLabs.a-star.edu.sg )

Agency for Science Technology and Research https://orcid.org/0000-0003-0349-1557

\section{Research Article}

Keywords: SARS-CoV-2, COVID-19, serological, S protein, antibodies, IgG subclasses, symptomatic, severity, cytokines

Posted Date: April 28th, 2021

DOI: https://doi.org/10.21203/rs.3.rs-432867/v1

License: (a) This work is licensed under a Creative Commons Attribution 4.0 International License. Read Full License 


\section{Abstract}

\section{Purpose}

COVID-19, caused by Severe Acute Respiratory Syndrome Coronavirus (SARS-CoV-2), has a wide disease spectrum ranging from asymptomatic to severe. While it is widely accepted that specific humoral immune responses are critical in controlling the infection, the relationship between the humoral immune response and disease severity is currently unclear.

\section{Methods}

Using a flow cytometry-based assay to detect specific antibodies against full length S protein, we compared the antibody levels between patients from different severity groups. We also analysed the cytokine profiles of patients from different severity groups by multiplex microbead-based immunoassay.

Results

We found an association between specific $\lg \mathrm{M}$, $\lg \mathrm{A}$ and $\lg \mathrm{G}$ against the spike protein and disease severity. By comparing the ratio of $\mathrm{Th} 1 \mathrm{lg} \mathrm{l} 1$ and $\lg \mathrm{G} 3$ to $\mathrm{Th} 2 \lg \mathrm{l} 2$ and $\lg \mathrm{G} 4$, we observed that all severity groups exhibited a ratio that was skewed towards a stronger Th1 response over Th2 response. In addition to the strong Th1 response, patients with severe disease also developed a Th2 response, as exemplified by the smaller ratio of $\lg \mathrm{G} 1$ and $\lg \mathrm{G} 3$ over $\operatorname{lgG} 2$ and $\operatorname{lgG} 4$ and the smaller Th1/Th2 cytokine ratios, compared to patients with mild disease severity.

\section{Conclusion}

The results suggest that acute severity or disease resolution is associated with a specific immunological phenotype. A smaller skew towards a Th1 response over Th2 response, during infection, may contribute to disease progression, while a greater skew towards a Th1 response over Th2 response may contribute to a better disease outcome. This may suggest potential therapeutic approaches to COVID-19 disease management.

\section{Introduction}

The coronavirus disease 2019 (COVID-19) is an on-going pandemic, affecting 223 countries, with 123 million confirmed human infection cases and more than 2 million fatalities to date [1]. The disease, caused by Severe Acute Respiratory Syndrome Coronavirus (SARS-CoV-2) [2-4], can lead to a series of clinical manifestations, ranging from mild to severe symptoms. Most patients have mild symptoms such as fever, cough, fatigue, anosmia, sore throat and headache [5, 6]. Approximately $20 \%$ of symptomatic patients develop severe disease, with $5 \%$ progressing to critical stages, which could include respiratory failure, pneumonia, multiple organ failure and, in the most serious cases, death $[5,7,8]$. It has been 
reported that spike protein (S protein)-specific humoral responses are particularly enriched in convalescent individuals, as compared to deceased individuals [9]. However, there is limited information on the role of antibodies in the modulation of disease severity. Similarly, while the immune responses in recovered COVID-19 patients following infection can persist for 6 months post infection [10], the immune longevity can vary considerably between individuals [11]. Thus, there is a critical need to better understand the humoral immunity to SARS-CoV-2 to inform public health decisions on vaccination, and, possibly, treatment and prophylaxis.

We have recently developed a flow cytometry assay based on full length S protein and found that IgG1 is the dominant IgG subclass in COVID-19 patients [12]. IgG1 and IgG3 induction, typically indicative of a T helper 1 (Th1) response [13], is a pro-inflammatory response particularly important in protective immunity against viruses. IgG1 and IgG3 possess higher neutralisation capabilities against many viruses [14-16]. On the other hand, IgG2 and IgG4, induced by a T helper 2 (Th2) response, have been hypothesised to mediate either viral infection enhancement or disease enhancement [17-19]. Similarly, the Th2 cytokine IL6 has been implicated as the trigger of a cytokine storm that could lead to clinical disease progression [20-22].

In this study, we examined the S protein antibody profiles in patients with asymptomatic SARS-Cov2 infection and with varying clinical severities of COVID-19. We also investigated whether disease severity was associated with IgG subclass imbalance and Th1/Th2 cytokine imbalance in COVID-19 patients. The longitudinal antibody profiles were also assessed to determine the longevity of the specific immune response. Understanding Th1/Th2 imbalance in SARS-Cov2 infection may provide insight into the pathogenic mechanisms and identify biomarkers of disease severity and new therapeutic approaches.

\section{Methods}

Plasma samples

COVID-19 patients

A total of 131 patients (symptomatic, $n=81$ and asymptomatic, $n=50$ ), who tested PCR-positive for SARSCoV-2 by nasopharyngeal swab, were recruited into the study from January to September 2020 [23]. Demographic data, clinical and laboratory parameters during the hospitalisation period are listed in Table S1 [24]. Symptomatic patients were classified into three groups based on clinical severity: mild (no pneumonia on chest radiographs [CXR] at baseline and during hospital admission), moderate (pneumonia on CXR without hypoxia), and severe (pneumonia on CXR with hypoxia [desaturation to $\leq 94 \%]$ ). Asymptomatic infections were individuals with a positive SARS-CoV-2 PCR test but without any reported symptoms attributable to COVID-19 in the 3 months before this first test till follow up 28 days later. To obtain plasma fractions, whole blood was collected from patients using BD Biosciences Vacutainer ${ }^{\circledR} \mathrm{CPT}^{\mathrm{TM}}$ tubes and centrifuged at $1700 \mathrm{~g}$ for $20 \mathrm{~min}$. Plasma samples were categorised by five time points: median 5 days post-illness onset (pio), median 10 days pio, median 23 days pio, median 101 days pio and median 180 days pio. 
A total of 20 individuals (Table S1), who were previously diagnosed with SARS-CoV during the outbreak in 2003 [25], were contacted and enrolled in the study. Plasma fractions were then isolated from the recovered SARS individuals. Archived human CoV patients' samples (Table S1) collected between 20122013 were also used in this study. This included samples from seven alpha-CoV (229E/NL63) and six beta-CoV (OC43) infections, which were confirmed using SeeGene RV12 respiratory multiplex kit [26].

\section{S protein flow cytometry-based assay for antibody detection (SFB assay)}

The assay was performed as previously described [12]. S protein-expressing cells were seeded at $1.5 \mathrm{x}$ $10^{5}$ cells per well in 96 well V-bottom plates (ThermoFisher Scientific). The cells were first incubated with human plasma (diluted 1:100 in 10\% FBS (HyClone) before a secondary incubation with a double stain, consisting of Alexa Fluor 647-conjugated secondary antibodies (diluted 1:500) and propidium iodide (PI (Sigma-Aldrich); diluted 1:2500). Secondary antibodies used are conjugated anti-human IgM, IgA or IgG (ThermoFisher Scientific). For assays examining IgG subclasses, the secondary incubation was with mouse anti-human IgG1 (ThermoFisher Scientific), IgG2 (BioLegend), IgG3 (BioLegend), or IgG4 (ThermoFisher Scientific). The cells were then incubated with Alexa Fluor 647-conjugated anti-mouse IgG (ThermoFisher Scientific). Cells were read on BD Biosciences LSR4 laser and analyzed using FlowJo (Tree Star). Cells were gated on: (1) FSC-A/SSC-A to exclude cell debris, (2) FSC-A/FSC-H for single cells, (3) FSC-A/PI for live cells (PI-negative population), (4) FITC/Alexa Fluor 647. Binding is determined by the percentage of GFP-positive $S$ protein-expressing cells that are bound by antibody, indicated by Alexa Fluor 647- and FITC-positive events.

\section{Multiplex Microbead-Based Immunoassay}

Plasma samples were treated with $\operatorname{Triton}^{\mathrm{TM}} \mathrm{X}-100$ (1\%) for virus inactivation [27]. Immune mediator levels were measured using Cytokine/Chemokine/Growth Factor 45-Plex Human ProcartaPlex ${ }^{\mathrm{TM}}$ Panel 1 (ThermoFisher Scientific, Waltham, MA, USA). Cytokine levels were also measured in 24 healthy donor plasma as baseline controls.

Analytes included granulocyte-macrophage colony-stimulating factor (GM-CSF), epidermal growth factor (EGF), brain-derived neurotrophic factor, beta-nerve growth factor (bNGF), basic fibroblast growth factor (FGF-2), hepatocyte growth factor (HGF), monocyte chemoattractant protein (MCP) 1, macrophage inflammatory protein (MIP) 1a, MIP-1b, RANTES (regulated on activation, normal T cell expressed and secreted), chemokine (C-X-C motif) ligand (CXCL) 1 (GRO-a), stromal cell-derived factor 1 (SDF-1a), interferon (IFN) gamma-induced protein 10 (IP-10), eotaxin, IFN-a, IFN-g, interleukin (IL) IL-1a, IL-1b, IL1RA, IL-2, IL-4, IL-5, IL-6, IL-7, IL-8, IL-9, IL-10, IL-12p70, IL-13, IL-15, IL-17A, IL-18, IL-21, IL-22, IL-23, IL-27, IL31, leukemia inhibitory factor (LIF), stem cell factor (SCF), tumor necrosis factor (TNF-a), TNF-b, vascular endothelial growth factors A and D (VEGF-A, VEGF-D), platelet derived growth factor (PDGF-BB), and placental growth factor (PLGF-1). 
Data were anlyzed on Bio-Plex Manager ${ }^{\mathrm{TM}}$ 6.1.1 (Bio-Rad Laboratories). Standard curves were generated with a 5-parameter logistic algorithm, reporting values for both MFI and concentration. Internal control samples were included in each Luminex assay to remove potential plate effects. Readouts of these samples were used to normalize the assayed plates. A correction factor was obtained from the differences across the multiple assays and this correction factor was then used to normalize all samples. The concentrations were logarithmically transformed to ensure normality. Samples with concentrations out of measurement range were assigned the value of logarithmic transformation of Limit of Quantification (LOQ).

\section{Statistical analysis}

Statistical analysis was done using Prism (GraphPad). For comparing between multiple groups, KruskalWallis tests and post hoc tests using Dunn's multiple comparison tests were used to identify significant differences. $P$-values less than 0.05 are considered significant, where * indicates $P \leq 0.05$, ** indicates $P \leq 0.01, \star \star \star$ indicates $P \leq 0.001, * \star \star \star$ indicates $P \leq 0.0001$.

\section{Results}

High levels of specific antibodies against $S$ protein are associated with disease severity

Using a flow cytometry-based assay to detect antibodies against full length $S$ protein [12], we first examined if specific IgM, IgA and IgG against the S protein were associated with COVID-19 disease severity ( $n=81$; Table S1) over the early course of infection, at time points of median 5 days, 10 days and 23 days post-illness onset (pio). The patients were classified into three groups: mild (no pneumonia), moderate (pneumonia with no hypoxia), or severe (pneumonia with hypoxia) [28]. At median 5 days pio, the $S$ protein antibody responses were low, regardless of the isotype (Fig. 1). We did not observe any significant difference between the three disease severity groups. At median 10 days and 23 days pio, $S$ protein $\lg M$, IgA and IgG responses were associated with disease severity (Fig. 1). Patients in the severe group had higher IgM, IgA and IgG responses at median 10 days and 23 days pio. Various groups of controls (Table S1) were also assessed in parallel (Fig. S1): (1) recovered SARS individuals ( $n=20),(2)$ healthy controls $(n=22)$, and (3) seasonal human CoV patients $(n=20)$.

We further examined if the S protein antibody responses were associated with clinical outcomes (Fig. S2) such as pneumonia, requirement for supplemental oxygen and intensive care unit (ICU) admission. S protein antibody responses were associated with pneumonia at median 23 days pio, while all three isotypes, IgM, IgA and IgG responses were associated with more severe clinical outcomes, requirement for supplemental oxygen and ICU admission, at a median of 10 days pio.

We proceeded to study if the disease severity was associated with a particular IgG subclass. At median 5 days pio, IgG subclass responses were low (Fig. 2). At median 10 and 23 days pio, where the IgG subclass responses were higher, we found an association between all IgG subclasses and the disease severity at both median 10 days and 23 days pio (Fig. 2). We also observed an association between all 
IgG subclasses and specific clinical outcomes such as pneumonia, requirement for supplemental oxygen and ICU admission (Fig. S3). Higher IgG1, IgG2, IgG3 and IgG4 responses were detected in patients with pneumonia at median of 23 days pio, but not at median of 10 days. In contrast, the association between higher responses for all IgG subclasses and requirement for supplemental oxygen was found as early as 10 days pio.

IgG subclass imbalance is associated with disease severity

We investigated whether disease severity was associated with IgG subclass imbalance. To this end, we combined the IgG1 and IgG3 responses, typically indicative of Th1 responses, and IgG2 and IgG4 responses, typically indicative of Th2 responses, and computed the ratio of the combined IgG1 and IgG3 response to the combined IgG2 and IgG4 responses,13/24 ratio (Fig. 3A). A ratio of 1 indicates a balanced IgG subclass response, where the combined IgG1 and IgG3 response is similar to the combined $\lg \mathrm{G} 2$ and $\lg \mathrm{G} 4$ response. A ratio higher than 1 indicates $\lg$ subclass imbalance, where the responses were skewed towards a more dominant $\lg \mathrm{G} 1$ and $\lg G 3$ response. Comparing the $13 / 24$ ratio across the different severity groups, we did not observe any association at the earlier time points, median 5 days and 10 days pio. However, at median 23 days pio, the 13/24 ratio was associated with disease severity, where the skew towards IgG1 and IgG3 Th1 response was greater in patients with mild disease than patients with moderate and severe disease. The mild group have a mean 13/24 ratio of 22.39 , compared with 1.56 and 1.38 in the moderate and severe groups, respectively. We also computed the ratio of the $\lg \mathrm{G} 1$ response to the IgG4 response, $1 / 4$ ratio (Fig. 3B). Similar to the 13/24 ratio, we found an association between the 1/4 ratio and disease severity. Interestingly, the association between the 1/4 ratio and disease severity was more pronounced - the $1 / 4$ ratio was significantly higher in the mild group (58.88) than the moderate group (5.88), which, in turn, was significantly higher than the severe group (2.36).

We then investigated if patients with no symptom also have an IgG subclass imbalance with a dominant IgG1 and IgG3 response. Asymptomatic patients ( $\mathrm{n}=50$; Table S1) developed specific antibodies, and IgG1 is the dominant IgG subclass response (Fig. $3 \mathrm{C}$ ). Indeed, the asymptomatic patients were found to have a IgG subclass imbalance skewed towards a dominant IgG1 and IgG3 response (mean 13/24 ratio: 75.41; mean 1/4 ratio: 252.1, Fig. 3D).

\section{Th1/Th2 cytokine imbalance is associated with disease severity}

To determine if the skewed Th1 over Th2 response observed with IgG subclasses was also evident in the cytokine profile, we examined the cytokine profiles for the three severity groups (Fig. S4A). We did not find any association between Th1 cytokines, IL-2 and TNF-a, and disease severity (Fig. S4B). Higher IFN-g levels, another Th1 cytokine, were found in the severe group, compared to the mild group, at median 23 days pio (Fig. S4A). In contrast, IL-6, a Th2 cytokine, was found to be higher in the severe group, compared with the mild and moderate group at median 5 days and 10 days pio (Fig. S4B). Interestingly, when we compared the ratio of IL-2 to IL-6, the IL-2/IL-6 ratio was the smallest with the severe group, compared with the mild and moderate groups, at median 5 days and 10 days pio (Fig. 4). Similarly, we also observed an association between the IFN-g/IL- 6 ratio and disease severity and an association 
between the TNF-a/IL- 6 ratio and disease severity at median 5 days and 10 days pio (Fig. 4). The severe group exhibited the smallest ratio $(4.9,140.7$ and 0.5 for IL-2/IL-6, IFN-g/IL-6 and TNF-a/IL-6 ratios respectively) at median 5 days pio, showing that the bias towards Th1 cytokines was much less pronounced in the severe group (compared to the mild and moderate groups). On the contrary, asymptomatic patients had ratios of $639.2,500.7$ and 119.1 for IL-2/IL-6, IFN-g/IL- 6 and TNF- $\mathrm{a} / \mathrm{IL}-6$ ratios respectively (Fig. S4C), exhibiting a more prominent Th1 over Th2 response.

\section{IgG subclass and cytokine imbalance over the course of infection}

To understand if IgG subclass imbalance was sustained over the course of infection, we examined the IgG subclass response at later stages, median 101 days and 180 days pio. At these later stages, all patients, including those in the severe group, have recovered from the disease. At both time points, all patients retained substantial IgG1 response, and IgG1 remained the dominant IgG subclass (Fig. S5). $\lg$ 2, IgG3 and IgG4 responses were low across all three severity groups at both time points. When we compared the 13/24 and the $1 / 4$ ratios across the three severity groups, the trend, where the ratio was higher with the mild severity group than the moderate and severe severity group, was still sustained at median 101 days pio (Fig. 5A and 5B). However, at median 180 days pio, there is no significant difference in the $13 / 24$ and $1 / 4$ ratios between the three severity groups.

Comparing between the different time points, we observed that both 13/24 and 1/4 ratios were the lowest at median 10 days pio for patients with mild symptoms (Fig. 5C and 5D). Patients with moderate and severe symptoms exhibited lowest 13/24 and 1/4 ratios at median 23 days pio. Both 13/24 and 1/4 ratios increased over the time points, suggesting that IgG subclass imbalance towards a greater Th1 dominance increased over the time points in line with clinical recovery.

We also looked at the cytokine profile of the patients at median 101 days and 180 days pio (Fig. S4A). All three IL-2/IL-6, IFN-g/IL-6 and TNF-a/IL-6 ratios were similar across the three severity groups (Fig. 6A). While we did not see any significant difference in the three ratios across the time points for the mild group, we observed that a lower IL-2/IL- 6 at earlier time points (median 5 and 10 days pio) and a higher IL-2/IL-6 ratio at later time points (median 23, 101 and 180 days pio) (Fig. 6B). For the severe group, the trend was more pronounced, with all three ratios being lower at earlier time points (median 5 and 10 days pio) and higher at later time points (median 23, 101 and 180 days pio). In general, severe patients demonstrated smaller skew towards Th1 over Th2 response at the early disease phase and the skew towards Th1 response gradually increased to similar levels as mild and moderate patients at the later time points in line with clinical recovery. The skew towards a greater Th1 over Th2 cytokine response at later time points is in agreement with our IgG subclass data, suggesting that a dominance of Th1 over Th2 responses might create a more favourable environment for clinical recovery.

\section{Discussion}

Clinical management of COVID-19 is largely based on supportive therapy to treat symptoms and corticosteroids to prevent respiratory failure. With the approval of COVID-19 vaccines by health agencies 
around the world, the spread of the disease is expected to slow. While host immune responses have been shown to offer protection against the disease, there is a need to better understand the underlying mechanisms leading to severe disease and the relationship between the host immune responses and disease severity.

In this study, using a flow cytometry assay based on the S protein [17], we found that specific antibody levels were strongly associated with disease severity, in line with other studies [24, 29, 30]. More specifically, a few studies have reported an association between $\lg G 1$ and $\lg G 3$ with severe disease and suggested that potential contributory role of $\lg \mathrm{G} 1$ and $\lg \mathrm{G} 3$ in severe disease progression [31-33]. However, in this study, we have observed an association between antibody levels and disease severity for all four IgG subclasses. More importantly, the comparison of the ratio of Th1 IgG1 and IgG3 to Th2 IgG2 and IgG4 (13/24) or the ratio of IgG1 to IgG4 (1/4) revealed that SARS-CoV-2 infections were associated with IgG subclass imbalance, where the IgG response was skewed towards Th1. Both ratios were associated with disease severity, where lower ratios were associated with severe disease. This highlights the complexity of the relationship between the humoral response and disease severity and suggests that an IgG subclass imbalance that was skewed towards Th1 over Th2 may be better at preventing severe disease. This was supported by the cytokine data, where the ratio of Th1 to Th2 cytokine (IL-2/IL-6, IFNg/IL-6 and TNF-a/IL-6) was lower in patients with severe disease, compared with patients with mild disease. Strong signals of Th2, rather than Th1, response has also been reported in patients with severe disease [34].

Following infection, it typically takes a few days before the host develops an antibody response. IgM is the usually the first isotype that is induced. Isotype switching occurs later, typically 1-3 weeks following infection. The need to take the time factor into consideration is evident in our data. While there was no difference in antibody levels across the three severity groups at median 5 days pio, we observed significant difference at later time points, median 10 days and 23 days pio. The differences in the antibody levels between the three severity groups were most pronounced at median 23 days pio. Unsurprisingly, it is only at median 23 days pio, where we observed a greater IgG subclass imbalance towards Th1 over Th2 response in the mild group. Interestingly, at median 23 days pio, there was an upward trend in the $\operatorname{lgG} 13 / 24$ and 1/4 ratio. At median 101 pio, the 13/24 and 1/4 ratios for all severity groups were greater, as compared with median 23 days pio, and the differences in 13/24 and 1/4 ratios between the severity groups were also less pronounced. At median 180 days pio, there were no significant differences in the IgG subclasses ratios between the severity groups. Similarly, the cytokine data showed that patients with severe symptoms had lower skew towards Th1 over Th2 response. This is also in line with another study [20], which studied the SARS-CoV-2 infection in ferrets and found low levels of type I and III interferons and high expression of IL- 6 at early time points. The authors observed that, as the infection progressed, the type I interferon response increased at later time points, coinciding with viral clearance. The authors postulated that severe symptoms stemmed from a dysregulated immune response and delayed induction of type I interferon expression [20,35]. The IL-6-induced cytokine storm stimulates a robust production of monocyte- and neutrophil-associated chemokines in patients with severe symptoms [20-22]. However, with the delayed induction of type I interferon response which may be 
crucial to disease control, the elevated Th2 response may contribute to severe disease progression. Our findings provided more support that, in our cohort where all patients eventually recovered from the disease, a response skewed towards Th1 over Th2 could be crucial to recovery. In the light of the recent publication by Combes et al. [36], it is possible that Th2 IgG2 and IgG4 may functionally block the production of mild disease-associated interferon-stimulated gene-expressing cells and dampen cellular responses to interferons. More studies would need to examine this further.

The IgG subclass imbalance was also found in asymptomatic patients. Both $13 / 24$ and $1 / 4$ ratios were high in asymptomatic patients, at levels comparable or higher than patients with mild symptoms. This further supported that a higher skewed IgG subclass imbalance towards Th1 over Th2 might be more favourable and a dominant Th1-associated IgG1 and IgG3 antibody response might be important in controlling symptom progression in COVID-19. Similarly, the ratio of Th1 to Th2 cytokine (IL-2/IL-6, IFN-

$\mathrm{g} / \mathrm{IL}-6$ and TNF- $\mathrm{a} / \mathrm{IL}-6)$ was comparable to the ratio observed with patients with mild symptoms. A recent study has also found that asymptomatic patients have a highly functional virus-specific immune response, comparable to symptomatic patients [37]. We have not performed direct comparison between the asymptomatic and symptomatic patients over time as the absence of symptoms in the asymptomatic patients makes it difficult to ascertain the date of illness onset.

\section{Conclusion}

Taken together, these findings demonstrated that SARS-CoV-2 infections led to IgG subclass imbalance, with a skew towards a Th1 over a Th2 response (Fig. 7). Interestingly, the IgG subclass imbalance was associated with disease severity, where Th1-dominant IgG1 and IgG3 responses were associated with milder disease outcome. A greater skew towards a Th2 response over Th1 response during the disease progression might contribute to a more severe disease outcome, while a greater skew towards a Th1 response over Th2 response might play a crucial role in controlling disease progression and clinical recovery (Fig. 7). This has clinical implications. COVID-19 vaccines that could induce a predominantly Th1 IgG1 and IgG3 antibody response might be better at controlling COVID-19 infections. Recent data from COVID-19 vaccine trials have shown that vaccinated individuals had milder disease [38-41]. Indeed, individuals vaccinated with COVID-19 vaccine, BNT162b1, have a Th1-dominant response [42]. In addition, in severe patients, where Th2 responses were higher, IL-6 receptor inhibitors, such as tocilizumab, might have therapeutic benefits in COVID-19 disease [43].

\section{Declarations}

\section{Acknowledgement}

The authors wish to thank Ding Ying and team for their help in patient recruitment, Dr Danielle Anderson and her team at Duke-NUS for their technical assistance, Dr Olaf Rotzschke and his team at the SIgN (A*STAR) Multiplex Analysis of Proteins (MAP) platform for their assistance in running multiplex 
microbead-based immunoassay and also to Dr Anis Larbi at SIgN for providing the healthy donor samples.

\section{Funding}

Biomedical Research Council (BMRC), the A*ccelerate GAP-funded project (ACCL/19-GAP064-R20H-H) from Agency of Science, Technology and Research (A`STAR), National Medical Research Council (NMRC) COVID-19 Research fund (COVID19RF-001, COVID-19RF-007, COVID-19RF-60) and A*STAR COVID-19 Research funding $(\mathrm{H} / 20 / 04 / \mathrm{g} 1 / 006)$.

\section{Conflict of Interests}

A patent application for the SFB assay has been filed (Singapore patent \#10202009679P: A Method Of Detecting Antibodies And Related Products). The authors declare no other competing interests.

\section{Data and materials availability}

All data are available upon request: Laurent Renia, ID labs and SIgN, renia_laurent@IDlabs.a-star.edu.sg

\section{Code availability}

Not applicable

\section{Authors' Contribution}

YSG conceptualised study, designed and conducted the experiments, analysed the data, and wrote the manuscript. SWF, SNA and CYPL designed and conducted the experiments, analysed the data, and wrote the manuscript. PXH conducted the experiments. BEY, MICC, PAT, SK, SP, SYT, LJS, YSL and DCL designed and supervised sample collection. LFPN and LR conceptualised study and wrote the manuscript. All authors revised and approved the final version of the manuscript.

\section{Ethics approval}

The study design and protocols for symptomatic and asymptomatic COVID-19, recovered SARS and seasonal human CoV patient cohorts were approved by National Healthcare Group Domain Specific Review Board and performed, following ethical guidelines in the approved studies 2012/00917, $2020 / 00091$ and 2020/00076 respectively. Healthy donor samples were collected in accordance with approved studies $2017 / 2806$ and NUS IRB 04-140. All studies were performed in accordance with the Declaration of Helsinki for Human Research.

\section{Consent to Participate}

Written informed consent was obtained from all participants.

\section{Consent to Publish}


The authors affirm that human research participants provided informed consent for publication of the data.

\section{References}

1. WHO. WHO Coronavirus Disease (COVID-19) https://www.who.int/emergencies/diseases/novelcoronavirus-20192021 [Accessed on Mar 24 2021]. Available from: https://www.who.int/emergencies/diseases/novel-coronavirus-2019.

2. Cohen J, Normile D. New SARS-like virus in China triggers alarm. Science. 2020;367(6475):234-5.

3. Wu F, Zhao S, Yu B, Chen YM, Wang W, Song ZG, et al. A new coronavirus associated with human respiratory disease in China. Nature. 2020;579(7798):265-9.

4. Zhou P, Yang XL, Wang XG, Hu B, Zhang L, Zhang W, et al. A pneumonia outbreak associated with a new coronavirus of probable bat origin. Nature. 2020;579(7798):270-3.

5. Chen N, Zhou M, Dong X, Qu J, Gong F, Han Y, et al. Epidemiological and clinical characteristics of 99 cases of 2019 novel coronavirus pneumonia in Wuhan, China: a descriptive study. Lancet. 2020;395(10223):507-13.

6. Yang X, Yu Y, Xu J, Shu H, Xia J, Liu H, et al. Clinical course and outcomes of critically ill patients with SARS-CoV-2 pneumonia in Wuhan, China: a single-centered, retrospective, observational study. Lancet Respir Med. 2020;8(5):475-81.

7. Wu Z, McGoogan JM. Characteristics of and Important Lessons From the Coronavirus Disease 2019 (COVID-19) Outbreak in China: Summary of a Report of 72314 Cases From the Chinese Center for Disease Control and Prevention. JAMA. 2020;323(13):1239-42.

8. Zhou F, Yu T, Du R, Fan G, Liu Y, Liu Z, et al. Clinical course and risk factors for mortality of adult inpatients with COVID-19 in Wuhan, China: a retrospective cohort study. Lancet. 2020;395(10229):1054-62.

9. Atyeo C, Fischinger S, Zohar T, Slein MD, Burke J, Loos C, et al. Distinct Early Serological Signatures Track with SARS-CoV-2 Survival. Immunity. 2020;53(3):524-32 e4.

10. Dan JM, Mateus J, Kato Y, Hastie KM, Yu ED, Faliti CE, et al. Immunological memory to SARS-CoV-2 assessed for up to 8 months after infection. Science. 2021;371(6529).

11. Chia WN, Zhu, F., Ong S.W. X., Young, B.E., Fong, S. W., Le Bert, N., Tan, C. W., Tiu, C., Zhang, J., Tan, S. Y., Pada, S., Chan, Y.H., Tham, C. Y. L., Kunasegaran, K., Chen, M. I. C., Low, J. G.H., Leo, Y. S., Renia, L. Bertoletti, A., Ng, L. F. P., Lye, D. C. Wang, L. F. Dynamics of SARS-CoV-2 neutralising antibody responses and duration of immunity: a longitudinal study. The Lancet Microbe. 2021; In press.

12. Goh YS, Chavatte JM, Lim Jieling A, Lee B, Hor PX, Amrun SN, et al. Sensitive detection of total antiSpike antibodies and isotype switching in asymptomatic and symptomatic individuals with COVID19. Cell Rep Med. 2021;2(2):100193.

13. Kawasaki Y, Suzuki J, Sakai N, Isome M, Nozawa R, Tanji M, et al. Evaluation of T helper-1/-2 balance on the basis of IgG subclasses and serum cytokines in children with glomerulonephritis. Am 
J Kidney Dis. 2004;44(1):42-9.

14. Hofmeister Y, Planitzer CB, Farcet MR, Teschner W, Butterweck HA, Weber A, et al. Human IgG subclasses: in vitro neutralization of and in vivo protection against West Nile virus. J Virol. 2011;85(4):1896-9.

15. Richardson SI, Lambson BE, Crowley AR, Bashirova A, Scheepers C, Garrett N, et al. IgG3 enhances neutralization potency and Fc effector function of an HIV V2-specific broadly neutralizing antibody. PLoS Pathog. 2019;15(12):e1008064.

16. Walker MR, Eltahla AA, Mina MM, Li H, Lloyd AR, Bull RA. Envelope-Specific IgG3 and IgG1 Responses Are Associated with Clearance of Acute Hepatitis C Virus Infection. Viruses. 2020;12(1).

17. Arvin AM, Fink K, Schmid MA, Cathcart A, Spreafico R, Havenar-Daughton C, et al. A perspective on potential antibody-dependent enhancement of SARS-CoV-2. Nature. 2020;584(7821):353-63.

18. de Alwis R, Chen S, Gan ES, Ooi EE. Impact of immune enhancement on Covid-19 polyclonal hyperimmune globulin therapy and vaccine development. EBioMedicine. 2020;55:102768.

19. Liu L, Wei Q, Lin Q, Fang J, Wang H, Kwok H, et al. Anti-spike IgG causes severe acute lung injury by skewing macrophage responses during acute SARS-CoV infection. JCI Insight. 2019;4(4).

20. Blanco-Melo D, Nilsson-Payant BE, Liu WC, Uhl S, Hoagland D, Moller R, et al. Imbalanced Host Response to SARS-CoV-2 Drives Development of COVID-19. Cell. 2020;181(5):1036-45 e9.

21. Cao X. COVID-19: immunopathology and its implications for therapy. Nat Rev Immunol. 2020;20(5):269-70.

22. Chua RL, Lukassen S, Trump S, Hennig BP, Wendisch D, Pott F, et al. COVID-19 severity correlates with airway epithelium-immune cell interactions identified by single-cell analysis. Nat Biotechnol. 2020;38(8):970-9.

23. Pung R, Chiew CJ, Young BE, Chin S, Chen MI, Clapham HE, et al. Investigation of three clusters of COVID-19 in Singapore: implications for surveillance and response measures. Lancet. 2020;395(10229):1039-46.

24. Amrun SN, Lee CY, Lee B, Fong SW, Young BE, Chee RS, et al. Linear B-cell epitopes in the spike and nucleocapsid proteins as markers of SARS-CoV-2 exposure and disease severity. EBioMedicine. 2020;58:102911.

25. Leong HN, Earnest A, Lim HH, Chin CF, Tan C, Puhaindran ME, et al. SARS in Singapore-predictors of disease severity. Ann Acad Med Singap. 2006;35(5):326-31.

26. Jiang L, Lee VJ, Cui L, Lin R, Tan CL, Tan LW, et al. Detection of viral respiratory pathogens in mild and severe acute respiratory infections in Singapore. Sci Rep. 2017;7:42963.

27. Darnell A, Rimola J, Belmonte E, Ripoll E, Garcia-Criado A, Caparroz C, et al. Evaluation of LI-RADS 3 category by magnetic resonance in US-detected nodules $</=2 \mathrm{~cm}$ in cirrhotic patients. Eur Radiol. 2021.

28. Wong JEL, Leo YS, Tan CC. COVID-19 in Singapore-Current Experience: Critical Global Issues That Require Attention and Action. JAMA. 2020;323(13):1243-4. 
29. Okba NMA, Muller MA, Li W, Wang C, GeurtsvanKessel CH, Corman VM, et al. Severe Acute Respiratory Syndrome Coronavirus 2-Specific Antibody Responses in Coronavirus Disease Patients. Emerg Infect Dis. 2020;26(7):1478-88.

30. Long QX, Liu BZ, Deng HJ, Wu GC, Deng K, Chen YK, et al. Antibody responses to SARS-CoV-2 in patients with COVID-19. Nat Med. 2020;26(6):845-8.

31. Yates JL, Ehrbar DJ, Hunt DT, Girardin RC, Dupuis A, Payne AF, et al. Serological Analysis Reveals an Imbalanced IgG Subclass Composition Associated with COVID-19 Disease Severity. medRxiv. 2020:2020.10.07.20208603.

32. Chakraborty S, Gonzalez J, Edwards K, Mallajosyula V, Buzzanco AS, Sherwood R, et al. Proinflammatory IgG Fc structures in patients with severe COVID-19. Nat Immunol. 2021;22(1):67-73.

33. Luo H, Jia T, Chen J, Zeng S, Qiu Z, Wu S, et al. The Characterization of Disease Severity Associated IgG Subclasses Response in COVID-19 Patients. Frontiers in Immunology. 2021;12(460).

34. Roncati L, Nasillo V, Lusenti B, Riva G. Signals of Th2 immune response from COVID-19 patients requiring intensive care. Ann Hematol. 2020;99(6):1419-20.

35. Lucas C, Wong P, Klein J, Castro TBR, Silva J, Sundaram M, et al. Longitudinal analyses reveal immunological misfiring in severe COVID-19. Nature. 2020;584(7821):463-9.

36. Combes AJ, Courau T, Kuhn NF, Hu KH, Ray A, Chen WS, et al. Global absence and targeting of protective immune states in severe COVID-19. Nature. 2021;591(7848):124-30.

37. Le Bert N, Clapham HE, Tan AT, Chia WN, Tham CYL, Lim JM, et al. Highly functional virus-specific cellular immune response in asymptomatic SARS-CoV-2 infection. J Exp Med. 2021;218(5).

38. Ramasamy MN, Minassian AM, Ewer KJ, Flaxman AL, Folegatti PM, Owens DR, et al. Safety and immunogenicity of ChAdOx $1 \mathrm{nCoV}-19$ vaccine administered in a prime-boost regimen in young and old adults (COV002): a single-blind, randomised, controlled, phase 2/3 trial. Lancet. 2021;396(10267):1979-93.

39. Baden LR, El Sahly HM, Essink B, Kotloff K, Frey S, Novak R, et al. Efficacy and Safety of the mRNA1273 SARS-CoV-2 Vaccine. N Engl J Med. 2021;384(5):403-16.

40. Mulligan MJ, Lyke KE, Kitchin N, Absalon J, Gurtman A, Lockhart S, et al. Phase I/II study of COVID19 RNA vaccine BNT162b1 in adults. Nature. 2020;586(7830):589-93.

41. Polack FP, Thomas SJ, Kitchin N, Absalon J, Gurtman A, Lockhart S, et al. Safety and Efficacy of the BNT162b2 mRNA Covid-19 Vaccine. N Engl J Med. 2020;383(27):2603-15.

42. Sahin U, Muik A, Derhovanessian E, Vogler I, Kranz LM, Vormehr M, et al. COVID-19 vaccine BNT162b1 elicits human antibody and TH1 T cell responses. Nature. 2020;586(7830):594-9.

43. Rosas IO, Brau N, Waters M, Go RC, Hunter BD, Bhagani S, et al. Tocilizumab in Hospitalized Patients with Severe Covid-19 Pneumonia. N Engl J Med. 2021.

\section{Figures}



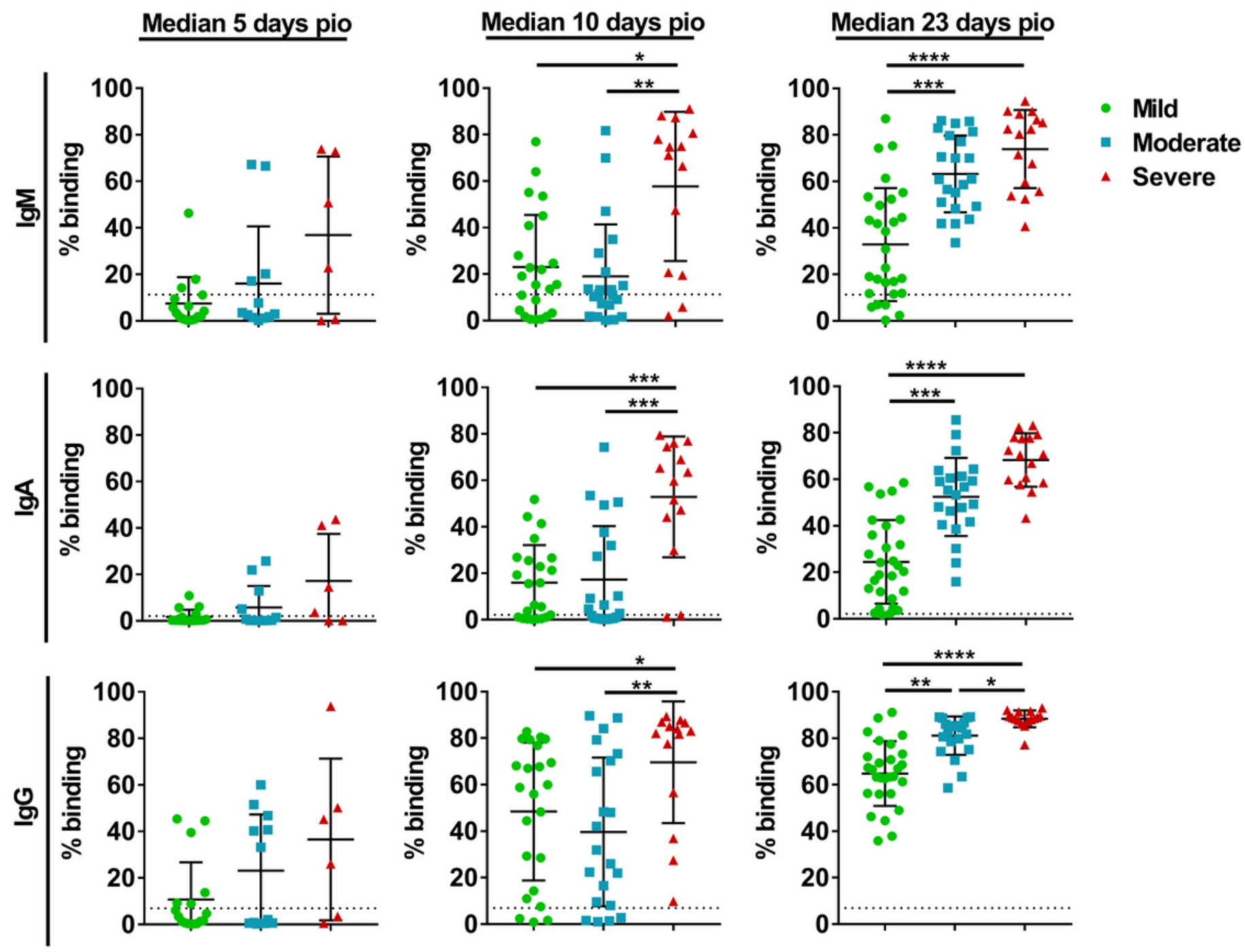

Figure 1

Specific antibodies against full length $S$ protein. Plasma samples were collected from symptomatic COVID-19 patients ( $n=81$ ) at time points median 5 days post-illness onset (pio) (mild, $n=17$; moderate, $n=12$; severe, $n=6), 10$ days pio (mild, $n=23$; moderate, $n=21$; severe, $n=14$ ) and 23 days pio (mild, $n=28$; moderate, $n=22$; severe, $n=16)$. Samples were screened at 1:100 dilution for specific $(A) \lg M,(B) \lg A$ and (C) IgG against full length SARS-CoV2 S protein expressed on the surface of HEK293T cells. Data are shown as mean \pm SD of two independent experiments. Statistical analysis was carried out using KruskalWallis tests, followed by post hoc Dunn's multiple comparison tests. P-values for comparisons between the three severity groups are shown, where * indicates $P \leq 0.05$, ** indicates $P \leq 0.01$, *** indicates $P \leq 0.001, \star \star \star \star$ indicates $P \leq 0.0001$. Dotted lines indicate mean $+3 S D$ of the healthy controls. 

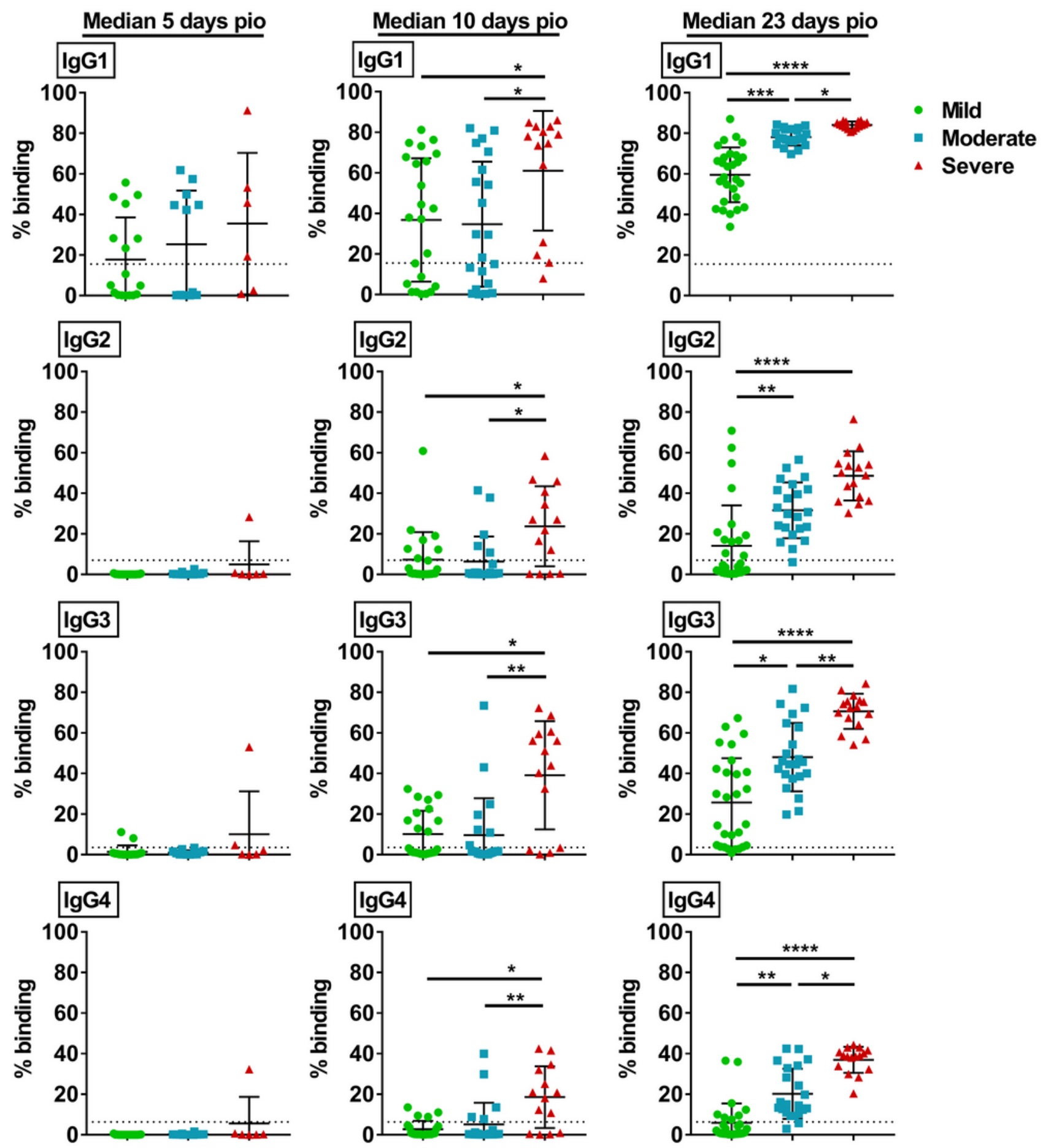

Figure 2

Specific IgG subclasses against full length $S$ protein. Plasma samples collected from 81 symptomatic COVID-19 patients at time points median 5 days pio (mild, $n=17$; moderate, $n=12$; severe, $n=6$ ), 10 days pio (mild, $n=23$; moderate, $n=21$; severe, $n=14$ ) and 23 days pio (mild, $n=28$; moderate, $n=22$; severe, $n=16$ ) were further screened for $\lg G$ subclasses, $\lg G 1, \lg G 2, \lg G 3$, and $\lg G 4$. Data are shown as mean \pm SD of two independent experiments. Statistical analysis was carried out using Kruskal-Wallis tests, followed by 
post hoc Dunn's multiple comparison tests. For (A-D), only P-values for comparisons between the three

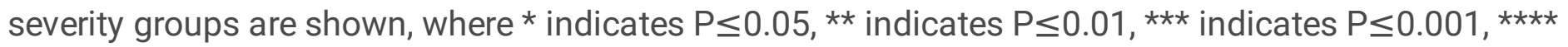
indicates $P \leq 0.0001$. Dotted lines indicate mean $+3 S D$ of the healthy controls.
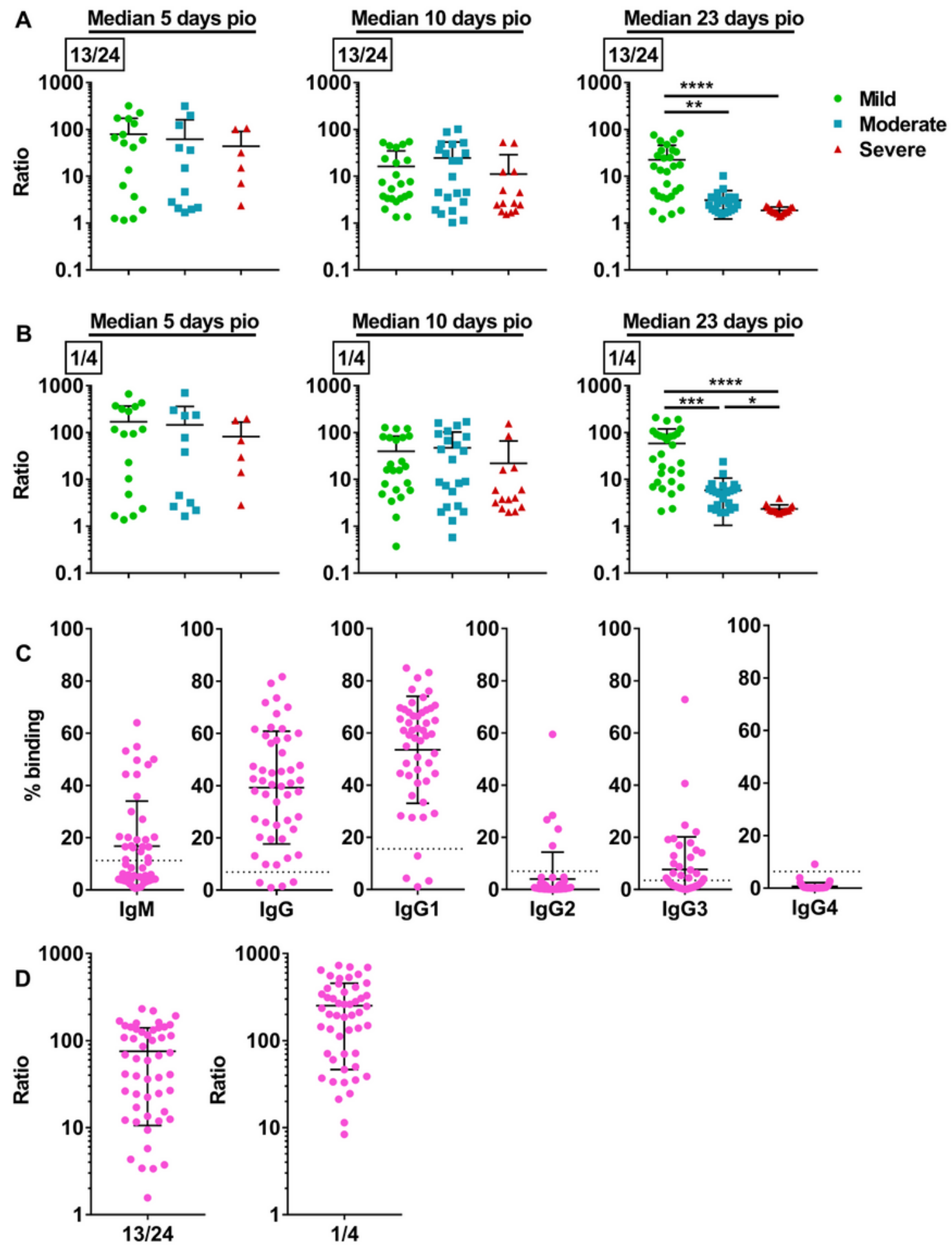

Figure 3

IgG subclasses imbalance in symptomatic and asymptomatic COVID-19 patients. IgG1, IgG2, IgG3, and IgG4 response in plasma samples collected from 81 symptomatic COVID-19 patients at time points 
median 5 days post-illness onset (pio) (mild, $n=17$; moderate, $n=12$; severe, $n=6$ ), 10 days pio (mild, $n=23$; moderate, $n=21$; severe, $n=14$ ) and 23 days pio (mild, $n=28$; moderate, $n=22$; severe, $n=16$ ) were further analysed. (A) Ratio of IgG1 response to IgG4 response, 1/4, and (B) ratio of combined IgG1 and IgG3 response to combined IgG2 and IgG4 response, 13/24, of the symptomatic patients are plotted. (C) Specific antibodies against full length $S$ protein in plasma samples collected from asymptomatic COVID19 patients $(n=50)$ on the day of positive PCR confirmation. Dotted lines indicate mean $+3 S D$ of the healthy controls. (D) Ratio of IgG1 response to IgG4 response, 1/4, and ratio of combined IgG1 and IgG3 response to combined IgG2 and IgG4 response, 13/24, of the asymptomatic patients are plotted. Data are shown as mean \pm SD of two independent experiments. Statistical analysis was carried out using KruskalWallis tests, followed by post hoc Dunn's multiple comparison tests. P-values for comparisons between the three severity groups are shown, where * indicates $P \leq 0.05$, ** indicates $P \leq 0.01$, *ᄎ* indicates $P \leq 0.001, * * * *$ indicates $P \leq 0.0001$. 
A
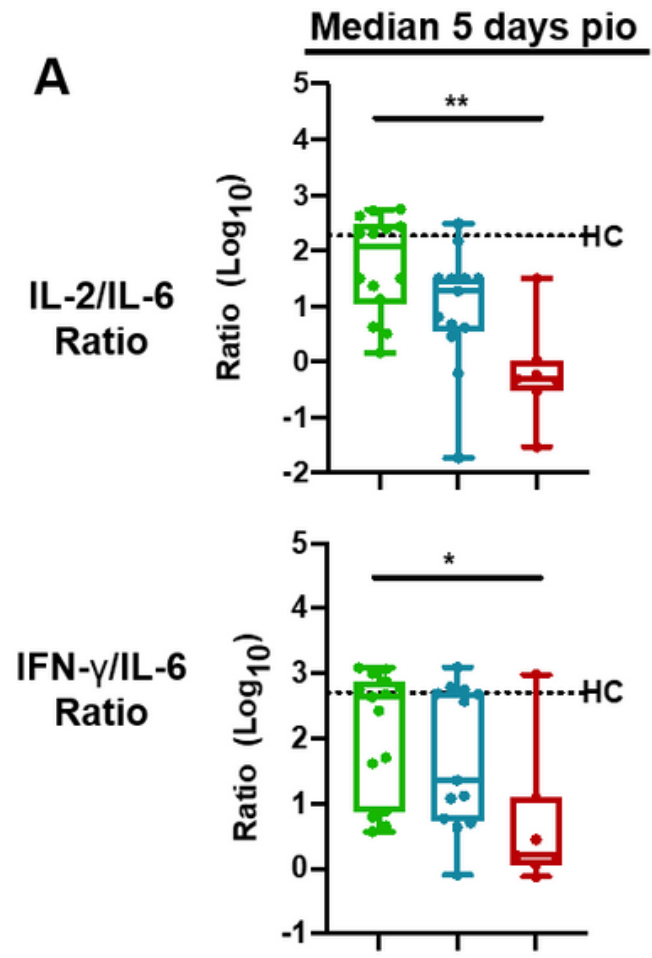

TNF- $\alpha /$ IL-6

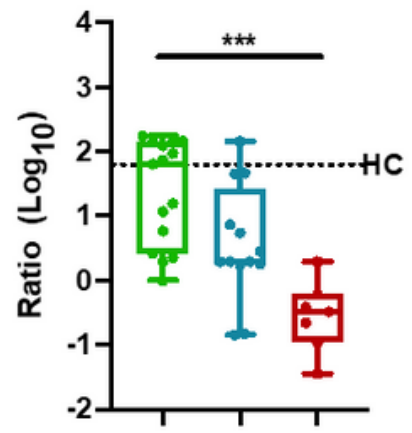

B
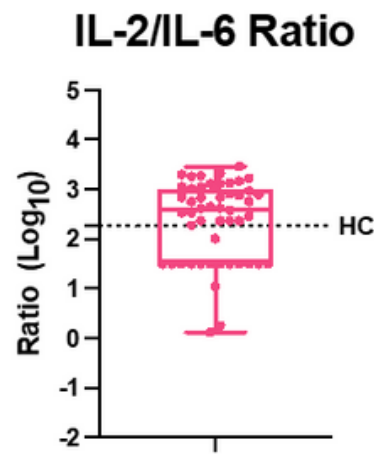

Mild
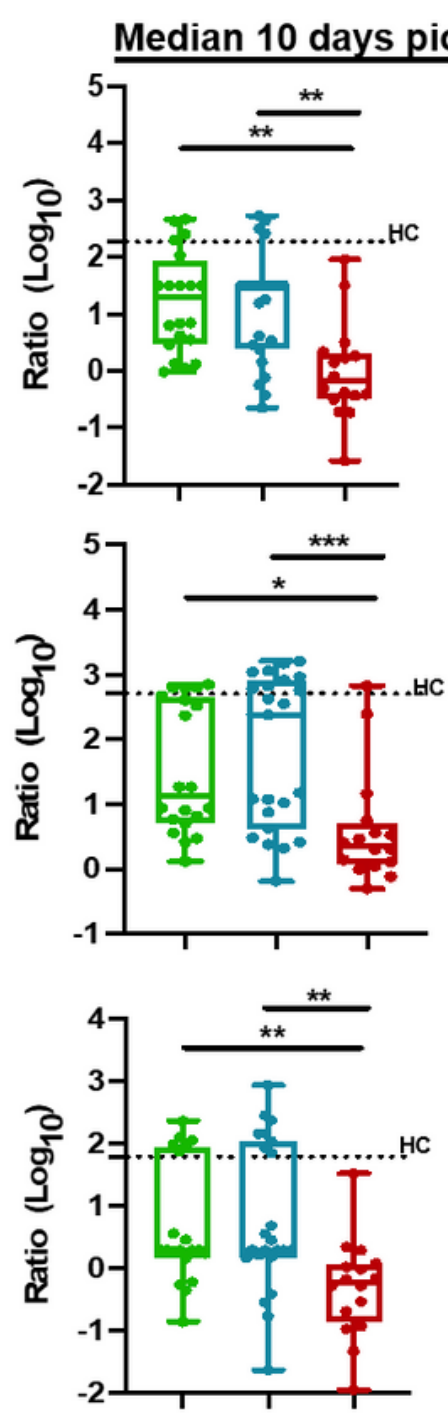

IFN-Y/IL-6 Ratio
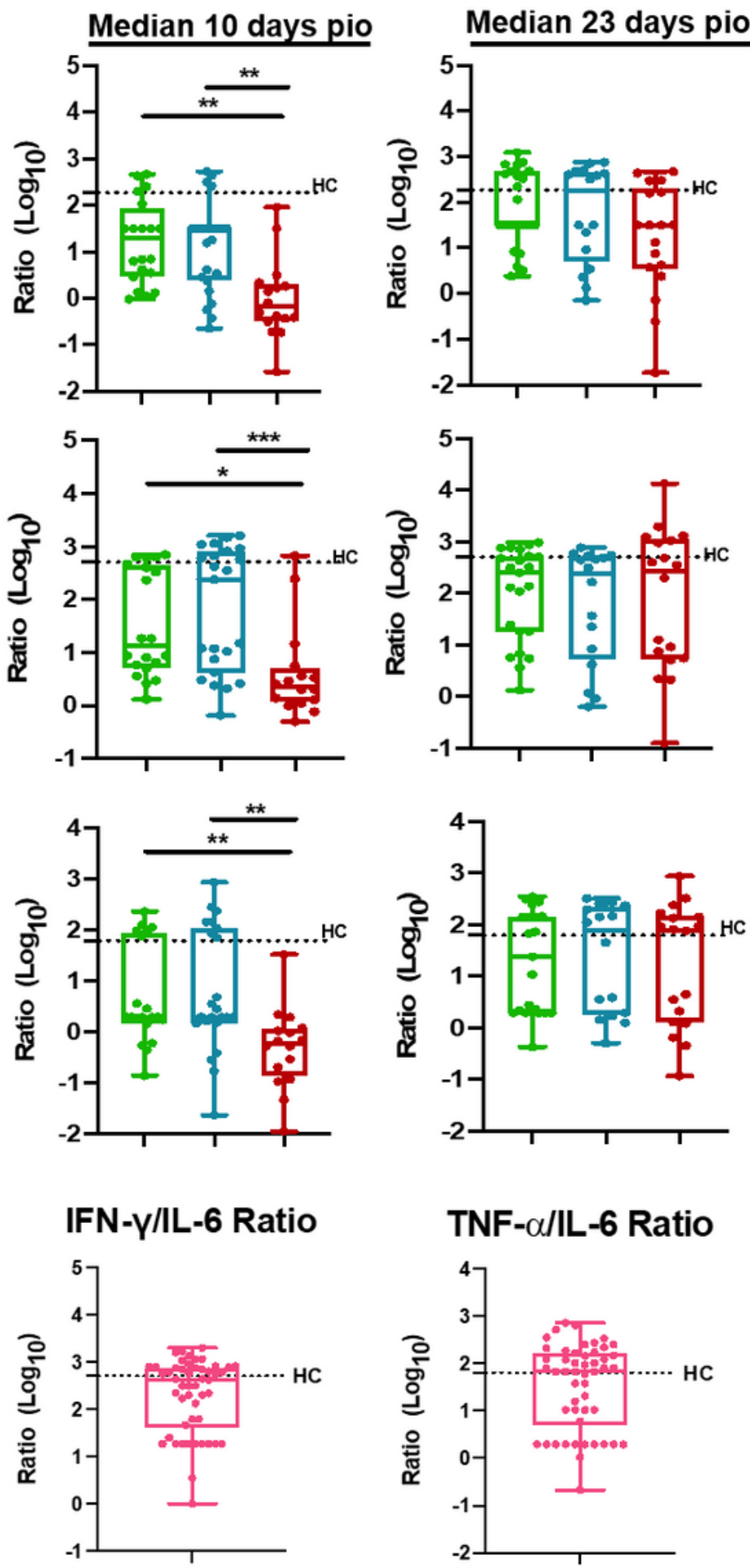

TNF- $\alpha /$ IL-6 Ratio

Severe

Asymptomatic

Figure 4

Th1/Th2 cytokine imbalance in symptomatic and asymptomatic COVID-19 patients. Plasma fractions were isolated from the blood of COVID-19 patients at different time points (median pio). Concentrations of Th1 (IL-2, IFN- $y$ and TNF-a) and Th2 (IL-6) cytokines were quantified using a 45-plex microbead-based immunoassay. (A) Th1/Th2 ratio in COVID-19 patients from different disease severity groups at median 5 days pio (mild, $n=14$; moderate, $n=13$; severe, $n=7$ ), 10 days pio (mild, $n=20$; moderate, $n=24$; severe, $n=15$ ) 
and 23 days pio (mild, $n=23$; moderate, $n=16$; severe, $n=18$ ) are illustrated as box plots. Kruskal-Wallis with Dunn's post hoc test was performed to discern the differences between the disease severity groups, where * indicates $P \leq 0.05$, ** indicates $P \leq 0.01$, *** indicates $P \leq 0.001$. (B) Th1/Th2 ratio in asymptomatic COVID-19 patients $(n=50)$ with active infection at median 2 days post-admission). Th1/Th2 cytokine ratio of healthy controls $(n=24)$ is indicated by the dotted line.
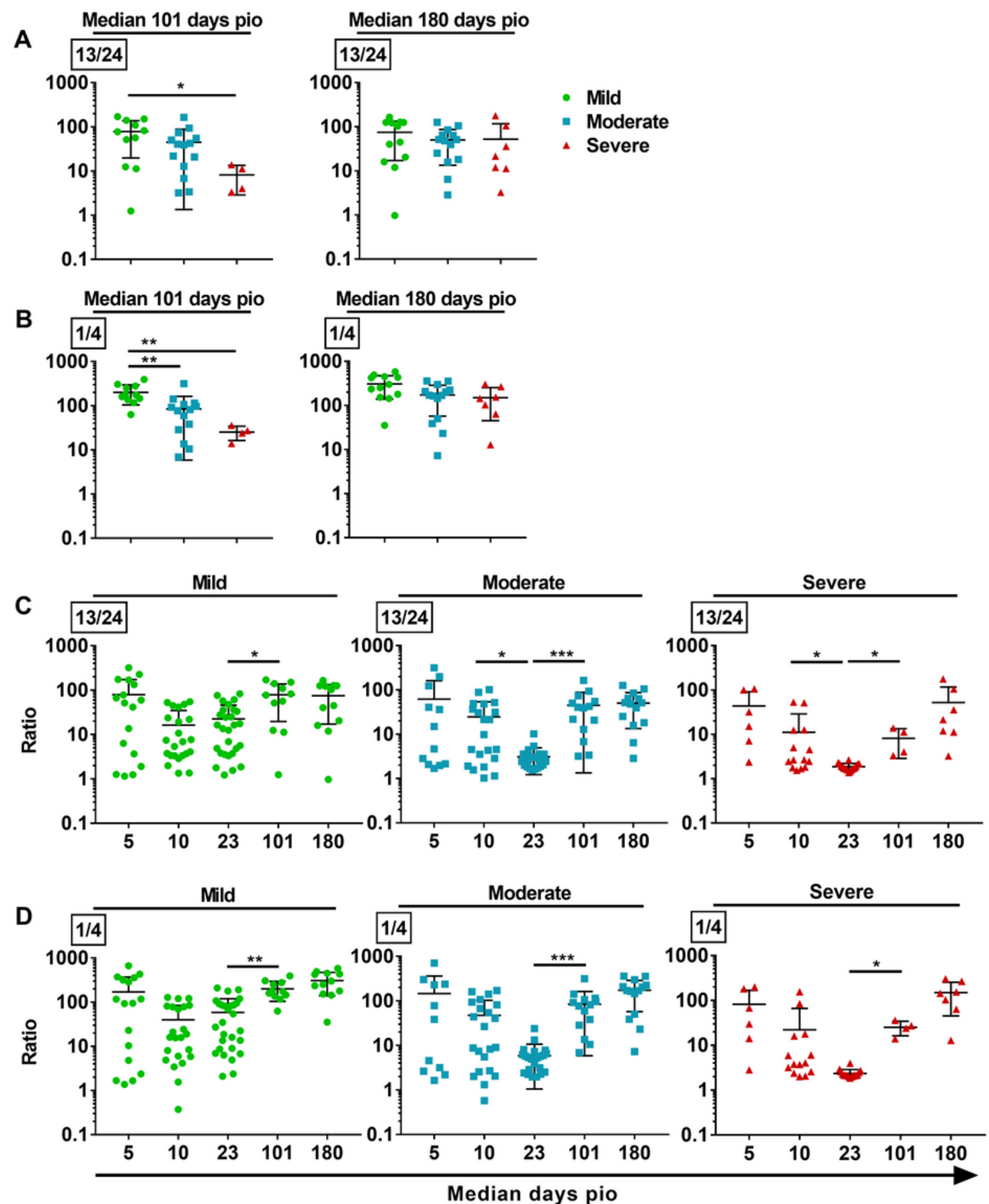

Figure 5 
Longitudinal examination of IgG subclasses imbalance in symptomatic and asymptomatic COVID-19 patients. IgG1, IgG2, IgG3, and IgG4 response in plasma samples collected from 81 symptomatic COVID19 patients at time points median 101 (mild, $n=11$; moderate, $n=14$; severe, $n=4$ ) and 180 (mild, $n=17$; moderate, $n=12$; severe, $n=6$ ) were further analysed. (A) Ratio of IgG1 response to IgG4 response, $1 / 4$, and (B) ratio of combined IgG1 and IgG3 response to combined IgG2 and IgG4 response, 13/24, of the symptomatic patients are plotted. Longitudinal profiles of (C) ratio of IgG1 response to IgG4 response, $1 / 4$, and (D) ratio of combined IgG1 and IgG3 response to combined IgG2 and IgG4 response, 13/24, up to median 180 days pio in COVID-19 patients, stratified by disease severity outcome. Data are shown as mean \pm SD of two independent experiments. Statistical analysis was carried out using Kruskal-Wallis tests, followed by post hoc Dunn's multiple comparison tests. P-values for comparisons between the three severity groups are shown, where * indicates $P \leq 0.05$, ** indicates $P \leq 0.01$, $\star \star \star$ indicates $P \leq 0.001$. 


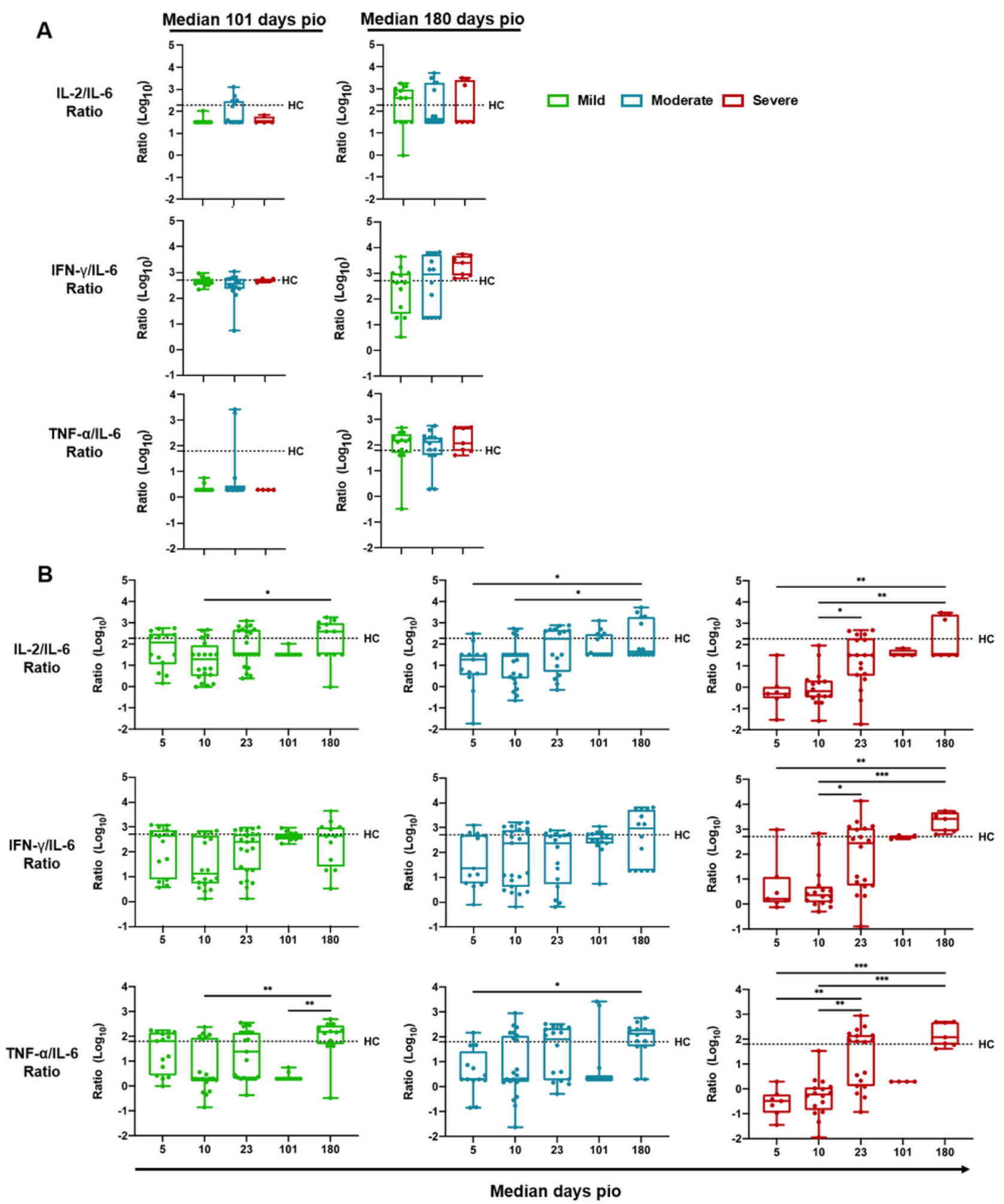

Figure 6

Dynamic changes of Th1/Th2 cytokine ratio among COVID-19 with different disease severity outcomes. Plasma fractions were isolated from the blood of COVID-19 patients at different time-points. Concentrations of Th1 (IL-2, IFN- $y$ and TNF-a) and Th2 (IL-6) cytokines were quantified using a 45-plex microbead-based immunoassay. (A) Th1/Th2 ratio in COVID-19 patients with different disease outcomes at median 101 days pio (mild, $n=11$; moderate, $n=14$; severe, $n=4$ ) and 180 days pio (mild, $n=12$; 
moderate, $n=14$; severe, $n=7$ ) are illustrated as box plots. Kruskal-Wallis with Dunn's post hoc test was performed to discern the differences between the disease severity groups. (B) Longitudinal profiles of Th1/Th2 ratio up to median 180 days pio in COVID-19 patients, stratified by disease severity outcome. Kruskal-Wallis with Dunn's post hoc test was performed to discern the differences across the five timepoints $\left({ }^{\star} p<0.05,{ }^{*} \mathrm{p}<0.01\right.$, and $\left.{ }^{* \star *} \mathrm{p}<0.001\right)$. Th1/Th2 cytokine ratio of healthy controls $(n=24)$ is indicated by the dotted line.

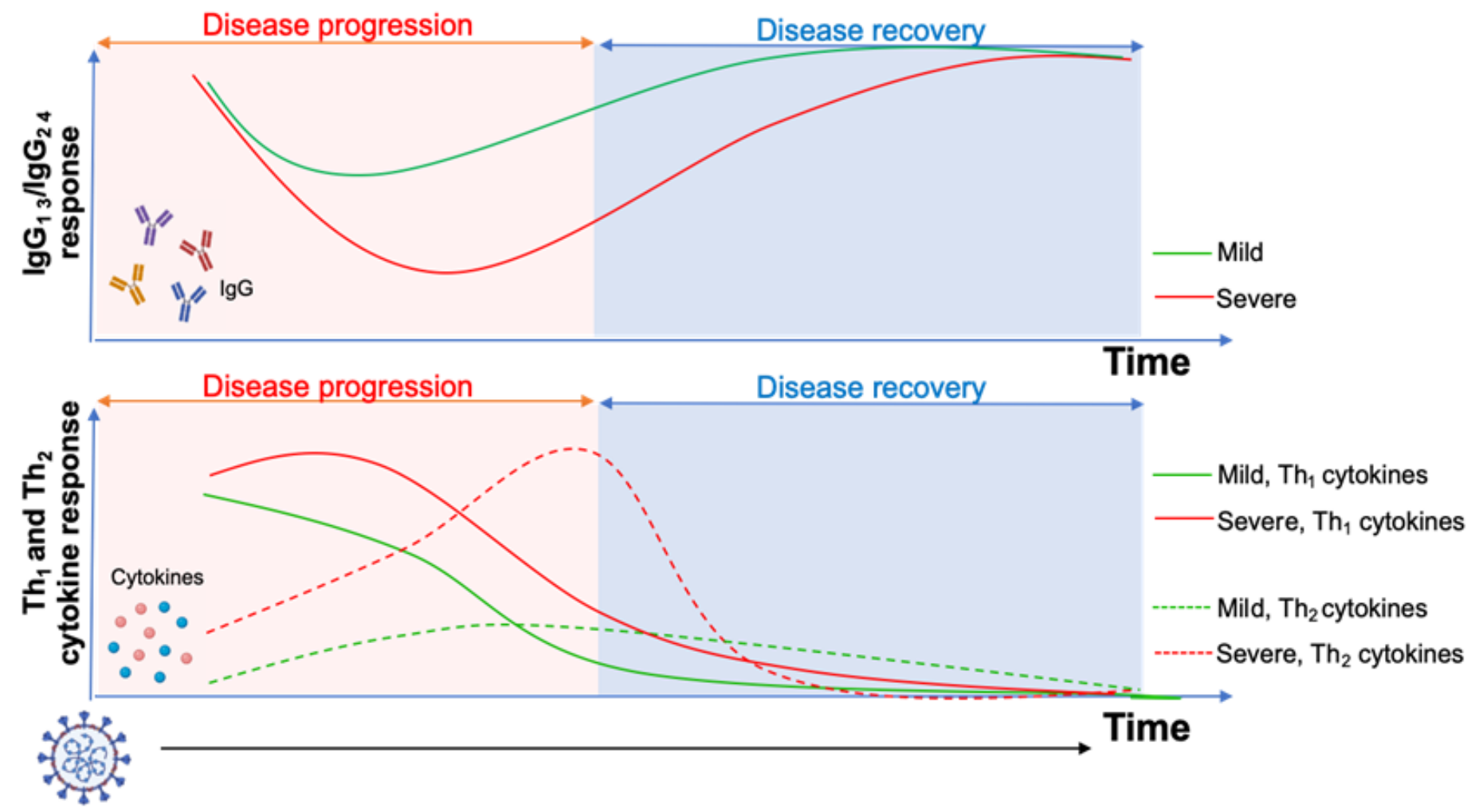

SARS-CoV-2 Infection

Early phase of disease progression

\section{$\mathrm{Th}_{2}$ dominant}

\section{$\mathrm{Th}_{1}$ dominant}

\section{Severity}

\section{Figure 7}

Dynamic alterations of anti-S-protein IgG subclass, and Th1 and Th2 cytokine responses in COVID-19. (Top panel) SARS-CoV-2 infections led to IgG subclass imbalance, where the IgG subclasses responses were skewed towards a Th1 over a Th2 response. Intriguingly, Th1-dominant IgG1 and IgG3 responses were associated with milder disease outcome. (Second panel) Severe COVID-19 patients showed high levels of Th1 and Th2 cytokines at the acute phase of SARS-CoV-2 infection. In contrast, Th1 cytokines were dominant in mild patients. (Bottom panel) A greater skew towards a Th2 response over Th1 
response during the disease progression might contribute to a more severe disease outcome while a greater skew towards a Th1 response over Th2 response during the disease progression be crucial for clinical recovery.

\section{Supplementary Files}

This is a list of supplementary files associated with this preprint. Click to download.

- Figs1.tif

- Figs2.tif

- FigS3.tif

- Figs4.tif

- FigS5.tif

- IsotypelmbalancetableS1JClinImmun14April2021.docx 